\title{
Laser vaporization of cervical intraepithelial neoplasia
}

\author{
MICHAEL R. STEVER, CPT, MC, USA \\ ENRIQUE HERNANDEZ, MAJ, MC, USA \\ KUNIO MIYAZAWA, COL, MC, USA
}

A proved protocol for the training of gynecology residents in laser vaporization of the cervix uteri is presented. During the study period, 105 women with cervical intraepithelial neoplasia underwent outpatient laser therapy performed by gynecology residents under the guidance and supervision of a gynecologic oncologist. Results are comparable to those reported in the literature by experienced laser therapists. The mean and median operative times were 10 and eight minutes, respectively. Seventyseven percent of the patients experienced only mild or no discomfort. The estimated blood loss was less than $1 \mathrm{~mL}$ in $88 \%$ of cases. Of patients with adequate follow-up evaluation, $91 \%$ were considered cured.

Vaporization of the cervix uteri via carbon dioxide laser has become an acceptable treatment of cervical intraepithelial neoplasia (CIN). Its advantages include precision, preservation of visibility of the transformation zone, and excellent cure rates. ${ }^{1-7}$ The disadvantages include production of smoke, bleeding, pain, longer treatment time, high instrument cost, and the need for special technical skills. ${ }^{1,8}$

This report reviews our experience in training gynecology residents in this laser technique. Study results regarding bleeding, pain, treatment time, and cure rates are presented and compared to others reported in the literature.

\section{Materials and methods}

In 1984, we established a protocol for training gynecology residents in the use of the carbon dioxide laser for treatment of CIN. In a 26-month period between 1984 and early 1987, 105 women underwent laser vaporization of the cervix uteri at our center. The procedure was performed by senior residents in gynecology under the guidance and direct supervision of a gynecologic oncologist who was experienced in this technique.
All residents were instructed individually about the biophysics of laser therapy, as well as about the safety measures to be observed when using this form of energy. ${ }^{9}$ They were given the opportunity to familiarize themselves with the equipment, which consisted of a Cavitron-300 laser coupled to a Zeiss colposcope. A 300-mm lens was used on the laser micromanipulator and a $400-\mathrm{mm}$ objective lens on the colposcope, resulting in a spot diameter of $2 \mathrm{~mm}$. Under supervision, the residents practiced their technique on an asbestos block. After mastering the technique, they were allowed to participate in the treatment of patients.

A woman evaluated in our clinic for CIN was a candidate for laser therapy under the following criteria: if the colposcopic examination was adequate (that is, if the transformation zone was visualized entirely and the upper limits of the lesions were seen); if the Papanicolaou smear, directed biopsy, and colposcopic impression agreed within one degree; and if the endocervical curettage was normal.

After obtaining informed consent, the procedure was performed. The supervisor outlined the outer limits of vaporization by firing the laser in bursts of $500 \mathrm{~ms}$ (Fig 1). With the laser on continuous



Fig 1. Lateral margins of vaporization marked with short laser bursts (original magnification, $\times 10$ ). 


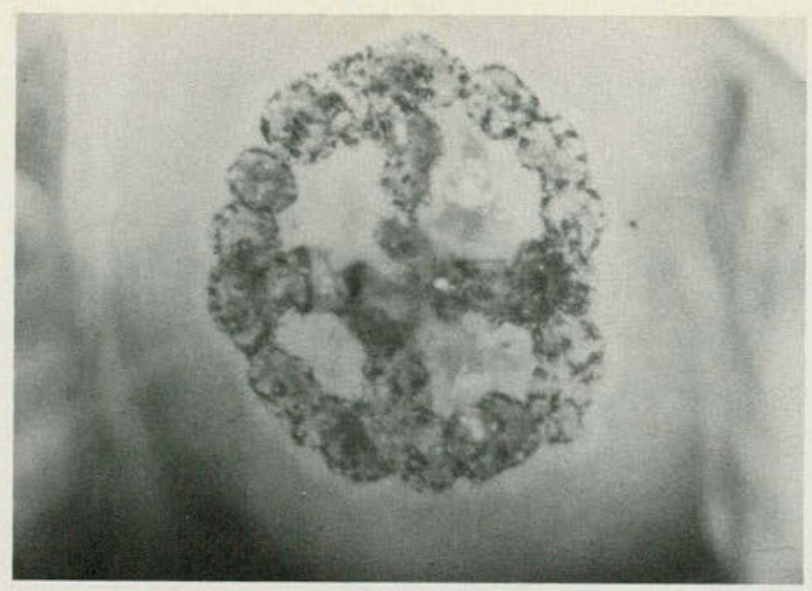

Fig 2. Transformation zone divided in quadrants (original magnification, $\times 10$ ).

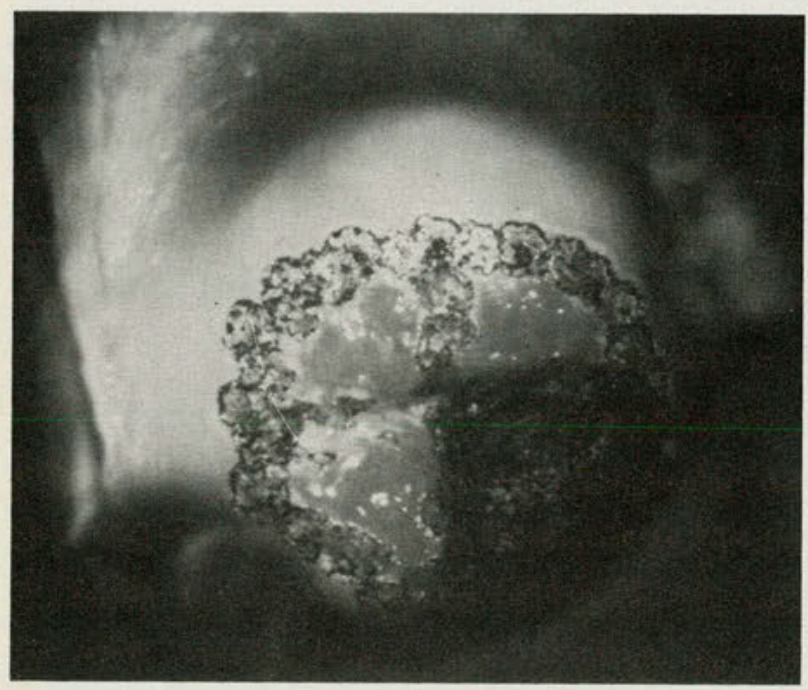

Fig 3. Left lower quadrant vaporized to depth of $7 \mathrm{~mm}$ (original magnification, $\times 10$ ).

mode and using the raster technique described by Bellina and associates, ${ }^{2}$ the transformation zone then was divided into quadrants (Fig 2) and vaporized one quadrant at a time (Fig 3). A power density of 700 to $800 \mathrm{~W} / \mathrm{cm}^{2}$ was used. The entire transformation zone was vaporized to a depth of 5 to 7 $\mathrm{mm}$, with a 2- to $3-\mathrm{mm}$ lateral margin.

All of the procedures were completed in the clinic setting. A smoke evacuator with filter was used. The patients received no medication before the procedure. Patients were asked to restrain from sexual intercourse, douching, and use of tampons for 14 days afterward. A follow-up appointment was scheduled for four weeks following treatment to document healing and complications. Thereafter, patients were evaluated every three months with
Papanicolaou smear and colposcopy, and directed biopsy if indicated.

For this report, a cure is defined as two normal Papanicolaou smears and colposcopic examinations performed four and seven months after laser therapy. All of the data presented was recorded prospectively.

\section{Results}

During the study period, 12 residents participated in the training protocol. Each resident performed 7 to 10 laser vaporizations. Their performance was judged satisfactory by the supervisor.

The mean age \pm SD of the 105 women treated was $26.5 \pm 6$ years. Forty-nine women were nulliparous. The racial distribution included 78 white, 14 black, and 13 oriental women. The diagnosis was CIN 1 in 47 patients (45\%), CIN 2 in 43 (41\%), and CIN 3 in 15 (14\%). The mean time \pm SD to complete the procedure was $10 \pm 7$ minutes; the median time was eight minutes.

During the procedure, 24 women $(23 \%)$ complained of moderate-to-severe "menstrual-like" cramps, which resolved soon after completing the procedure. Forty-five women $(43 \%)$ complained of mild cramps or of a feeling of "hot needles." Thirtysix patients $(34 \%)$ experienced no pain. A paracervical block was required to complete the procedure for five women who were experiencing severe pain. After anesthesia, the procedures were completed uneventfully and without discomfort. Two apprehensive patients were given paracervical block before initiating the vaporization. One experienced no pain; the other complained of mild cramps.

The estimated blood loss was less than $1 \mathrm{~mL}$ in 93 instances ( $88 \%$ ), between 1 and $5 \mathrm{~mL}$ in six cases $(6 \%)$, between 6 and $10 \mathrm{~mL}$ in four women $(4 \%)$, and between 11 and $15 \mathrm{~mL}$ in two cases (2\%). No women required immediate suturing to control bleeding. Monsel's solution was applied to the laser wound in several women. One patient returned bleeding one week after the procedure and required hemostatic stitching.

At the time of this writing, follow-up Papanicolaou testing and colposcopy has been performed for 57 women. The remaining 10 are not yet due for follow-up or have received military transfer. The transformation zone was completely visualized in 50 instances (88\%). Two follow-up Papanicolaou smears and colposcopic examinations are available for 35 patients. Only three $(9 \%)$ of these women had persistent CIN. Of the 22 women with only one follow-up visit, 21 had a normal Papanicolaou smear. In one, the cervical cytology showed atypical squamous cells, while colposcopic examination 
was adequate and no lesions were seen.

\section{Discussion}

Laser vaporization of the cervix uteri in properly selected patients with CIN now is a widely accepted treatment modality. Cure rates higher than $90 \%$ have been reported. ${ }^{3,6,10}$ At our teaching hospital, the laser treatment was performed by gynecology residents without increase in negative effects or decrease in cure rates when compared to the results reported by experienced laser therapists.

The average time required to perform a laser treatment in our clinic was 10 minutes. Popkin ${ }^{6}$ and Baggish ${ }^{10}$ reported average operative times of 6 to 8 minutes, respectively. Bellina and associates $^{3}$ reported operative times of 4 to 20 minutes.

Intraoperative pain was experienced by $66 \%$ of our patients. In only five women was the pain severe enough to require anesthesia (paracervical block), which resulted in resolution of their discomfort. Towsend and Richart ${ }^{7}$ reported that all 100 women treated by laser vaporization in their study experienced some pain. In 90 , the sensation was described as "tolerable discomfort"; six experienced severe cramps. Of the 138 women treated by Popkin, ${ }^{6}$ three ( $3 \%$ ) experienced severe pain, 82 $(59 \%)$ moderate pain, and $53(38 \%)$ minimal or no pain. Reporting on 156 patients, Caglar and coworkers $^{5}$ noted that $17(11 \%)$ experienced severe cramps. It has been postulated ${ }^{3}$ that the menstrual-cramp type of discomfort experienced during laser therapy is secondary to the release of prostaglandins. Prostaglandin-synthesis inhibitors and local anesthesia (paracervical or intracervical) have been used successfully in relieving patient discomfort. $1,3,8,10$

Of the 105 women in the current series, none experienced significant bleeding at the time of the procedure. However, one patient did return one week later because of bleeding and a hemostatic stitch was placed. Therefore, our data on intraoperative and postoperative bleeding compare favorably to that in the literature. Anderson ${ }^{1}$ reported laser vaporization could not be completed because of bleeding in one of his series of 543 patients. This woman received a hemostatic stitch, and the procedure was finished with diathermy. Six of his patients experienced significant delayed bleeding. Vaginal packing was used in five instances and diathermy in one.

Of the 100 women treated by Baggish, ${ }^{10}$ three required hemostatic suturing during the procedure. Another five experienced delayed bleeding; one of them required hemostatic stitching, and Monsel's solution was applied to the oozing wound of the other four. In Caglar and coworkers' ${ }^{5}$ series of 156 patients, laser vaporization could not be completed because of excessive bleeding in one case, and conization was performed. Another woman required suturing to control bleeding during the procedure. Significant delayed bleeding requiring treatment was experienced by six patients. Only one of the 100 women treated by Towsend and Richart ${ }^{7}$ required cauterization for delayed bleeding, and, of the 138 women treated by Popkin, ${ }^{6}$ none required treatment for bleeding.

Only Baggish ${ }^{10}$ quantitated the intraoperative blood loss. He reported an average loss of $10 \mathrm{~mL}$, with a range of 0 to $200 \mathrm{~mL}$. The estimated blood loss in $88 \%$ of our patients was less than $1 \mathrm{~mL}$, and none lost more than $15 \mathrm{~mL}$. It has been suggested $^{5,10}$ that excessive bleeding at the time of laser vaporization is associated with the presence of severe cervicitis, and that preoperative antibiotics are helpful in decreasing the blood loss in these patients. We did not assess the presence of cervicitis in our patients, and preoperative antibiotics were not used. Differences in patient populations, with a higher prevalence of cervicitis in the patients treated by Baggish, ${ }^{10}$ may explain the difference in blood loss reported by him and that observed by us.

Cryosurgery is very popular in the treatment of CIN because of its low cost, ease of application, and few complications. The cure rates are similar to those achieved with the laser. ${ }^{7,8}$ However, the transformation zone is visible in a significantly larger number of women following laser therapy. Jobson and Homesley ${ }^{11}$ reported that the transformation zone of $86 \%$ of their patients was visible following laser vaporization, compared to $52 \%$ following cryosurgery. The transformation zone was at the cervical portio or anatomic os in $87 \%$ of the 131 women treated by Burke. ${ }^{4}$ In the current series, $88 \%$ of

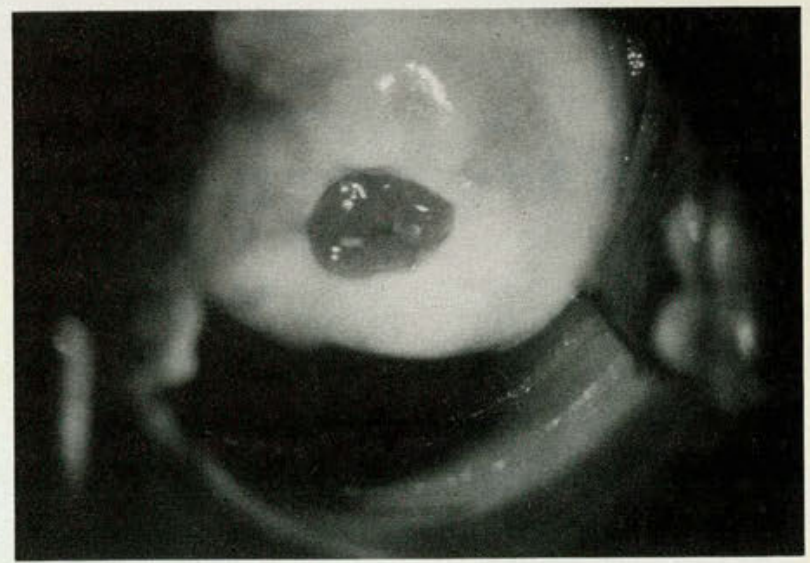

Fig 4. Healed cervix at four months after laser vaporization. The squamocolumnar junction is easily visible (original magnification, $\times 10$ ). 
the women examined four months after laser treatment had a visible transformation zone (Fig 4). This allows subsequent adequate colposcopic evaluations and conservative treatment for persistent or recurrent CIN

\section{Summary}

A protocol for the training of gynecology residents in the use of the carbon dioxide laser for the treatment of CIN is presented. This training was performed without any significant increase in operative time or complications or any decrease in cure rates.

The opinions and assertions contained herein are the private views of the authors and are not to be construed as official or as reflecting the views of the Department of the Army or the Department of Defense.

\footnotetext{
1. Anderson MC: Treatment of cervical intraepithelial neoplasia with the carbon dioxide laser: Report of 543 patients. Obstet Gynecol 1982;59:720-725.

2. Bellina JH, Wright, VC, Voros JI, et al: Carbon dioxide laser management of cervical intraepithelial neoplasia. Am J Obstet Gynecol 1981;141:828-832.
}

3. Bellina JH, Ross LF, Voros JI: Colposcopy and the $\mathrm{CO}_{2}$ laser for the treatment of cervical intraepithelial neoplasia: An analysis of seven years' experience. J Reprod Med 1983;28:147-151.

4. Burke L: The use of the carbon dioxide laser in the therapy of cervical intraepithelial neoplasia. Am J Obstet Gynecol 1982;144:337-340.

5. Caglar H, Ayhan A, Hreshchyshyn $\mathrm{MM}: \mathrm{CO}_{2}$ laser therapy for cervical intraepithelial neoplasia. Gynecol Oncol 1985;22:46-50.

6. Popkin DR: Treatment of cervical intraepithelial neoplasia with the carbon dioxide laser. Am J Obstet Gynecol 1983;145:177-180.

7. Towsend DE, Richart RM: Cryotherapy and carbon dioxide laser management of cervical intraepithelial neoplasia: A controlled comparison. Obstet Gynecol 1983;61:75-78.

8. Ferenczy A: Comparison of cryo- and carbon dioxide laser therapy for cervical intraepithelial neoplasia. Obstet Gynecol 1985;66:793-798.

9. Council on Scientific Affairs, American Medical Association: Lasers in medicine and surgery. JAMA 1986;256:900-907.

10. Baggish MS: A comparison between laser excisional conization and laser vaporization for the treatment of cervical intraepithelial neoplasia. Am J Obstet Gynecol 1986;155:39-44.

11. Jobson VW, Homesley HD: Comparison of cryosurgery and carbon dioxide laser ablation for treatment of cervical intraepithelial neoplasia, abstract. Obstet Gynecol Surv 1985;40:703-704.

From the Department of Obstetrics and Gynecology, Tripler Army Medical Center, Honolulu, Hawaii.

Reprint requests to Dr Hernandez, Medical College of Pennsylvania, 3300 Henry Ave, Philadelphia 19129. 